\title{
Measurement of Exciton and Trion Energies in Multistacked hBN/WS 2 Coupled Quantum Wells for Resonant Tunneling Diodes
}

\author{
Myoung-Jae Lee, ${ }^{*}{ }^{\dagger}$ David H. Seo, ${ }^{\perp}$ Sung Min Kwon, ${ }^{\ddagger}$ Dohun Kim,,${ }^{\S}$ Youngwook Kim, ${ }^{\S}$ \\ Won Seok Yun, ${ }^{\dagger}$ Jung-Hwa Cha, ${ }^{\dagger}$ Hyeon-Kyo Song, ${ }^{\top}$ Shinbuhm Lee, ${ }^{\S}$ MinKyung Jung, ${ }^{\dagger}$ \\ Hyeon-Jun Lee, ${ }^{\dagger}$ June-Seo Kim, ${ }^{\dagger}$ Jae-Sang Heo, ${ }^{\|}$Sunae Seo, ${ }^{\Uparrow}$ Sung Kyu Park, ${ }^{*}{ }^{\dagger}$
}

${ }^{+}$Convergence Research Institute, Daegu Gyeongbuk Institute of Science and Technology (DGIST), Daegu 42988, Korea

${ }^{\perp}$ PSK Inc., Hwasung 18449, Korea

${ }^{\ddagger}$ School of Electrical and Electronic Engineering, Chung-Ang University, Seoul 06974, Korea

${ }^{\S}$ Department of Emerging Materials Science, Daegu Gyeongbuk Institute of Science and Technology (DGIST), Daegu 42988, Korea

"Department of Physics, Sejong University, Seoul 05006, Korea

"Department of Medicine, University of Connecticut School of Medicine, Farmington, Connecticut 06030, United States

*E-mail for M.-J.L.: myoungjae.lee@ dgist.ac.kr.

*E-mail for S.K.P.: skpark@cau.ac.kr. 


\section{$\underline{\text { I. Characterizations of monolayer WS }} 2$ and hBN layer}

Figure $\mathrm{S} 1$ shows the PL image and spectrum of a monolayer $\mathrm{WS}_{2}$.

a

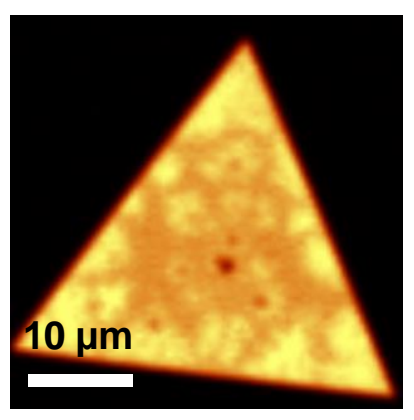

b

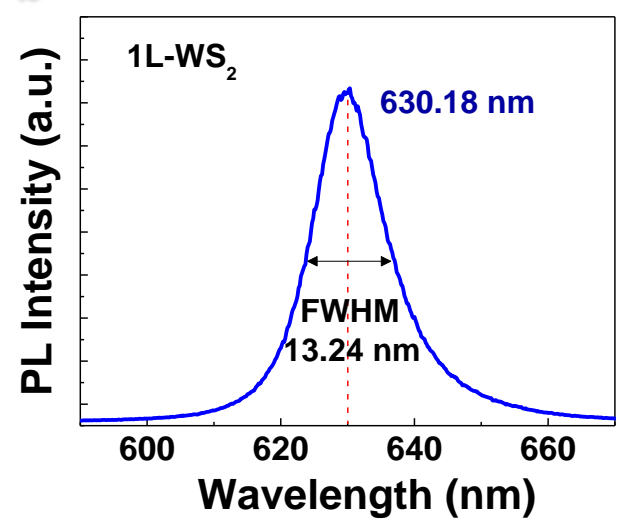

Figure S1. PL characterizations of monolayer $\mathrm{WS}_{2}$. (a) PL intensity spatial distribution image Scale bar, $10 \mu \mathrm{m}$. (b) PL spectrum of $\mathrm{WS}_{2}$ monolayer with its characteristic emission centers at a wavelength of $630.18 \mathrm{~nm}$ or binding energy of $1.97 \mathrm{eV}$ with a full-width at half maximum (FWHM) of $13.24 \mathrm{~nm}$ demonstrates the good quality of our monolayer sample. 
Figure S2 shows the SEM, AFM images, Raman spectrum and X-ray photoelectron spectroscopy (XPS) of a hBN. XPS was applied to determine the elemental stoichiometry of the synthesized h-BN. 2D hBN has been used extensively as an insulator for 2D heterostructures composed of various types of 2D semiconductors (e.g. WS $2, \mathrm{WSe}_{2}, \mathrm{MoS}_{2}$ etc).

a

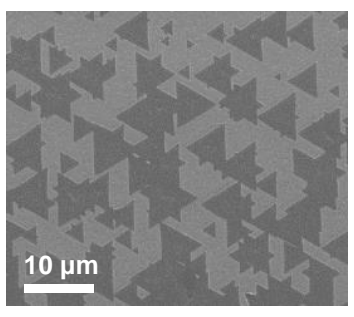

b

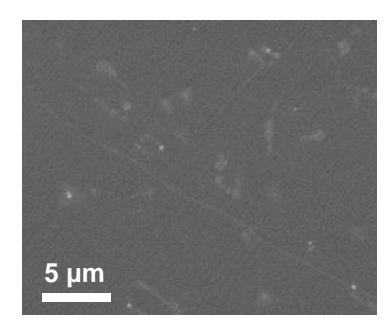

c

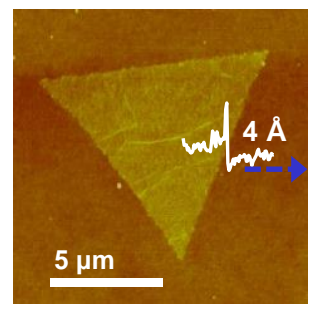

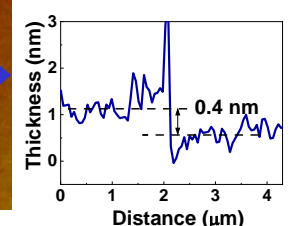

d

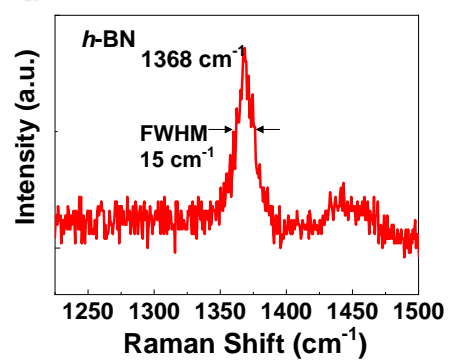

e

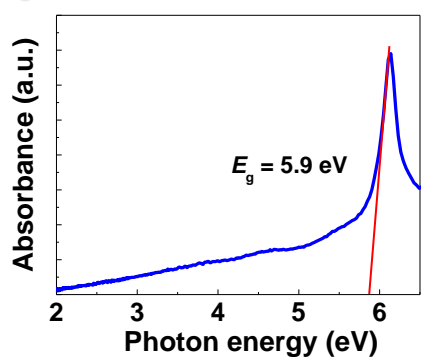

f

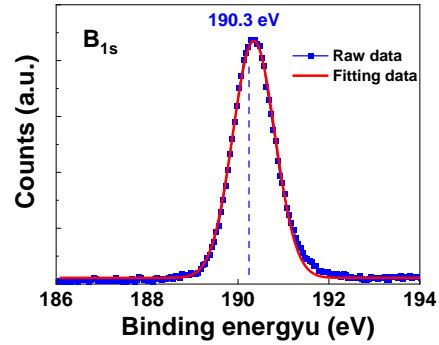

9

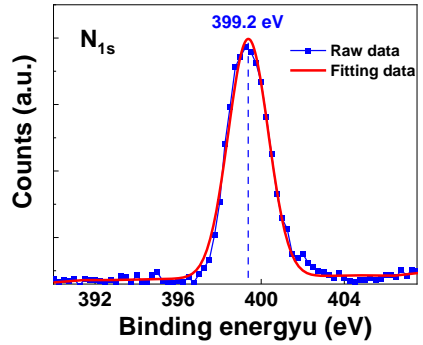

Figure S2. SEM, AFM, Raman, bandgap and XPS characterizations of hBN. (a-c) SEM and AFM images of (a) mono- or bilayers hBN with triangular morphologies, (b) large area bilayers hBN with full substrate coverage and (c) monolayer hBN, $0.4 \mathrm{~nm}$ thickness. (d) Raman spectrum of bilayer hBN with a peak at $1368 \mathrm{~cm}^{-1}$ originating from the $\mathrm{E}_{2 \mathrm{~g}}$ tangential mode of B-N bond inplane vibration with FWHM $15 \mathrm{~cm}^{-1}$. (e) Absorbance measurement by using UV spectrometer shows that $\mathrm{hBN}$ is an insulator with band gap $\sim 5.9 \mathrm{eV}$. (f-g) XPS spectra of h$\mathrm{BN}$, showing binding energies of $\mathrm{B}_{1 \mathrm{~s}}(190.3 \mathrm{eV})$ and $\mathrm{N}_{1 \mathrm{~s}}(392.2 \mathrm{eV})$, respectively. These binding energies are directly related with the hexagonal B-N bonding, implying the hexagonal phase of the BN film. 
a

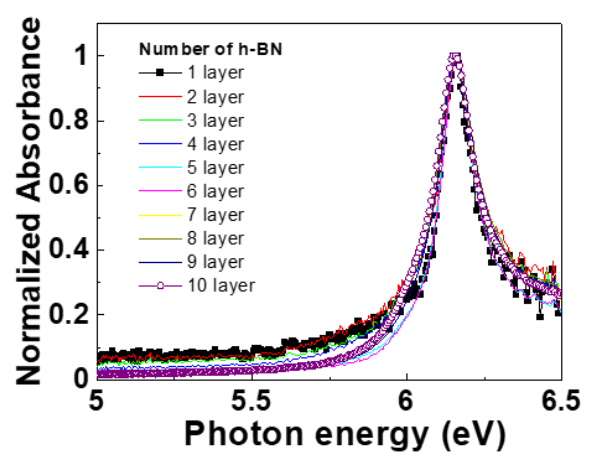

b

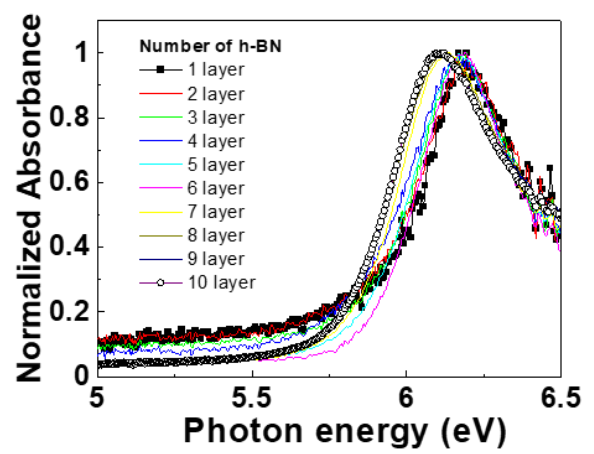

c

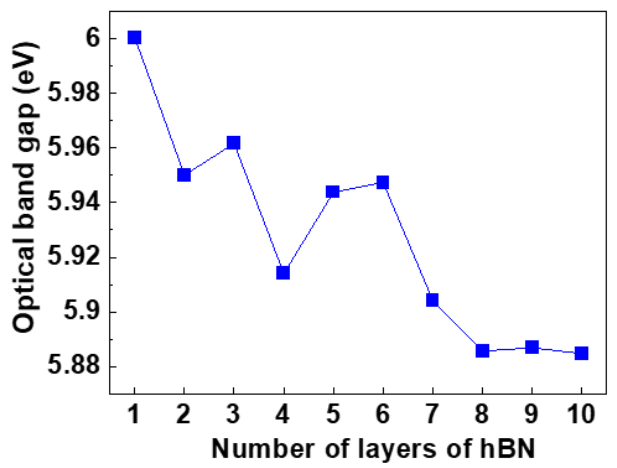

Figure S3. Layer number dependent absorption of h-BN. For up to 10 layers of transferred hBN (a) 10 samples as transferred. (b) 10 samples after $500{ }^{\circ} \mathrm{C}$ annealing for 3 hours in $\mathrm{H}_{2}$ to remove any contamination in between layers, (c) optical band gap $v s$ number of $\mathrm{hBN}$ layers for samples shown in (b) after around 8 layers and up to 10 layers $\mathrm{hBN}$ optical bandgap saturates at $5.88 \mathrm{eV}$. 
a

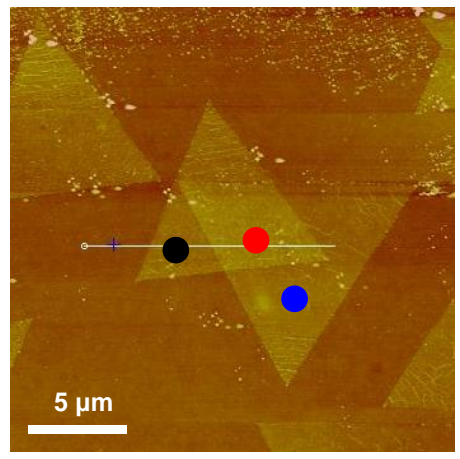

b

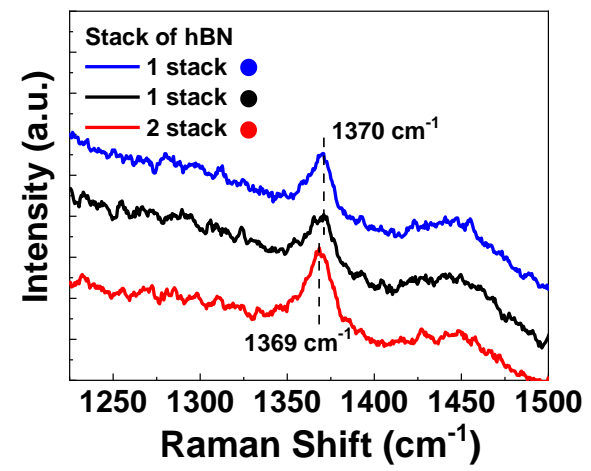

Figure S4. (a) AFM image of stacked hBN layer. Circles indicate positions for the Raman measurements. (b) Raman spectra with the corresponding position of (a) for 1 stack- (blue and black) and 2 stack-hBN (red) layers. 


\section{Stack Process of hBN/WS}

Multilayer stacks of $\mathrm{hBN} / \mathrm{WS}_{2}$ offer the prospect of creating a new class of heterostructure materials. By fine-tuning the number of the stacks, it may be possible to create tunable band gap with novel optical properties. We integrated a multistacked hBN/WS 2 CQWs structures, creating $\mathrm{WS}_{2}$ quantum wells sandwiched between alternated hBN layers. Investigations on van der Waals heterostructures are offering several new insights into low dimensional materials as film and sample quality begin to improve. In CQWs structure, an exciton is composed from an electron and a hole located within the same QW or across neighboring QWs in multistacked structures.

a
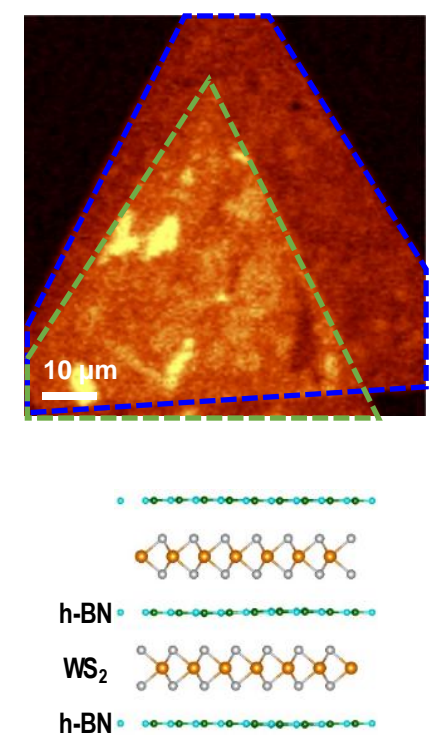

b
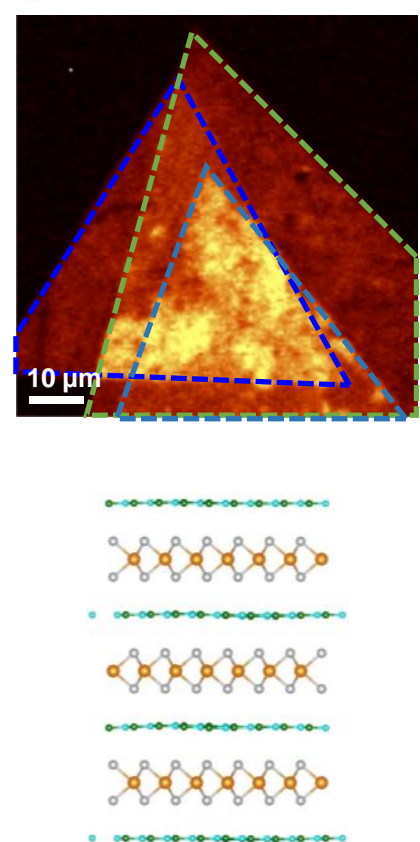

Figure S5. PL of hBN/WS 2 stacked. PL Intensity spectrum for (a) double-stacked hBN/WS 2 and (b) triple-stacked $\mathrm{hBN} / \mathrm{WS}_{2}$ 


\section{Simulation of the Stacked hBN/WS}

Since the interlayer van der Waals interaction has a crucial role in structural and electronic properties of sparse systems, we focused on the binding energy and the band structure of $\mathrm{WS}_{2}$ on and between h-BN layers. Based on the first-principles calculations within the generalized gradient approximation (GGA), we have examined the evolution of the QWsdependent electronic band structure of the $\mathrm{BN} / \mathrm{WS}_{2} / \mathrm{BN}(1 \mathrm{QW})$. In general, it is known that GGA calculations underestimate the bandgaps of semiconductors compared with their experimental values. However, they are highly powerful for predicting the trends of bandgap changes. Here, we performed an electronic band-structure calculation as a function of number of QWs and interlayer distance. As shown in Fig. 2b, we can observe the bandgaps from 1.8 to $1.72 \mathrm{eV}$ with increasing the thickness of QW. In addition, this tendency corresponds well to the experimental result. In addition, this tendency corresponds well to the experimental result observed in Figure $2 b$. 

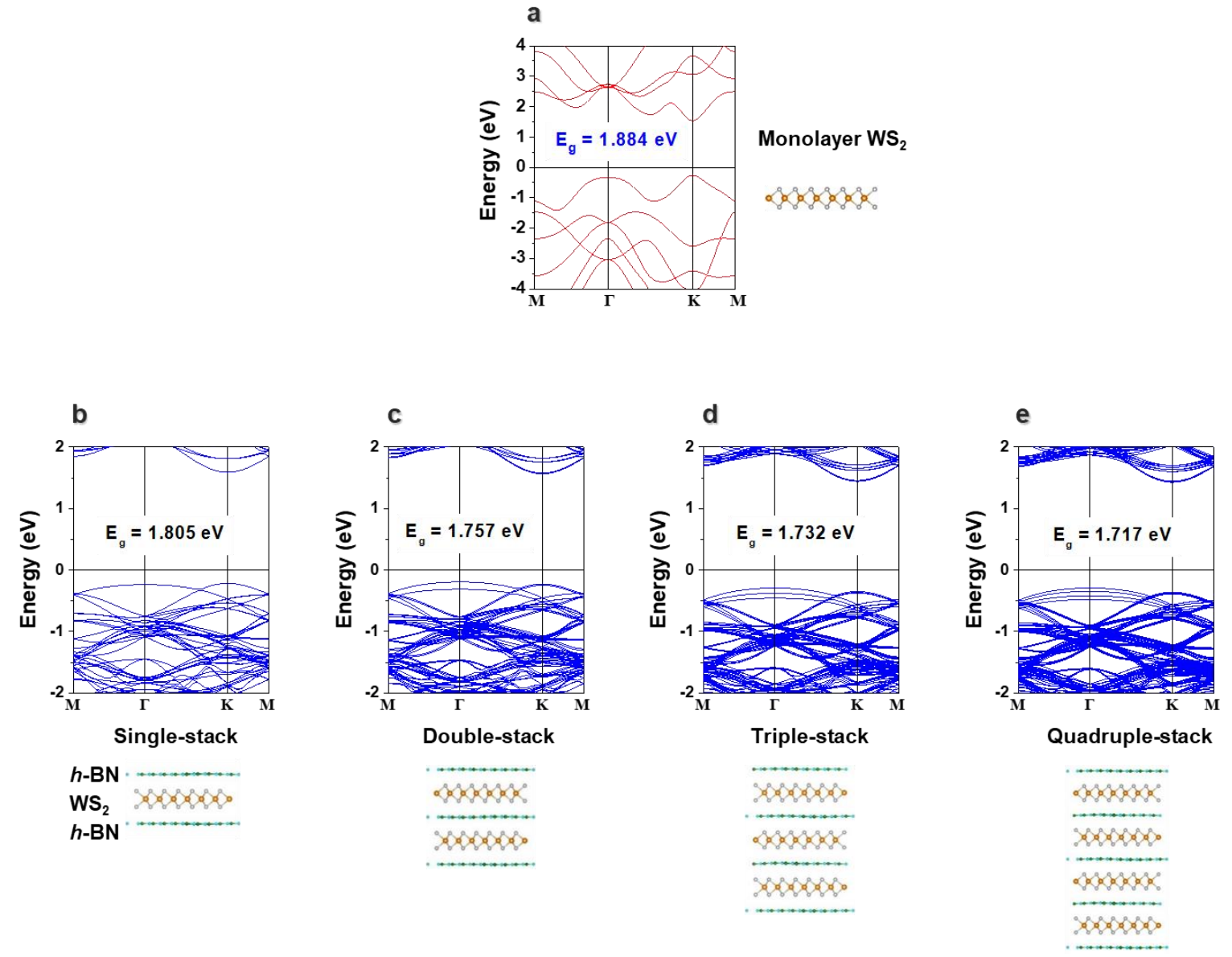

Figure S6. Electronic band structure of monolayer $\mathrm{WS}_{2}$ and $\mathrm{hBN} / \mathrm{WS}_{2}$ stacked structure. (a) $1 \mathrm{~L}-\mathrm{WS}_{2}$ and (b-e) from single-stacked to quadruple-stacked structures obtained from DFT calculations. 


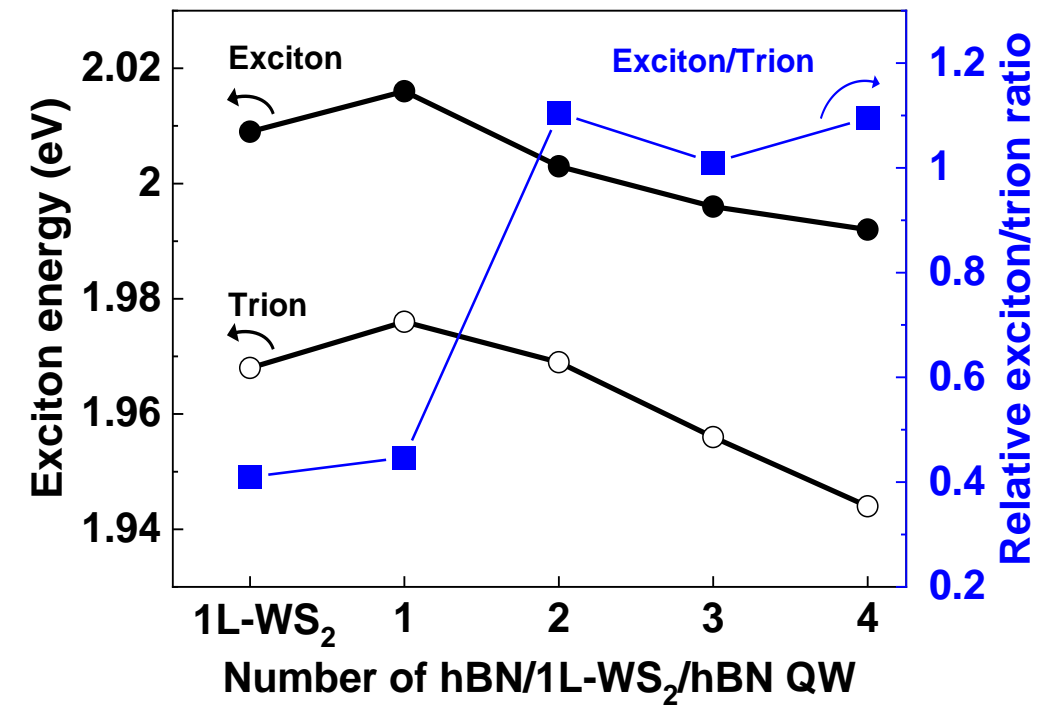

Figure S7. Comparison of the exciton and trion energy extracted from the PL spectra in Figure 2c. 

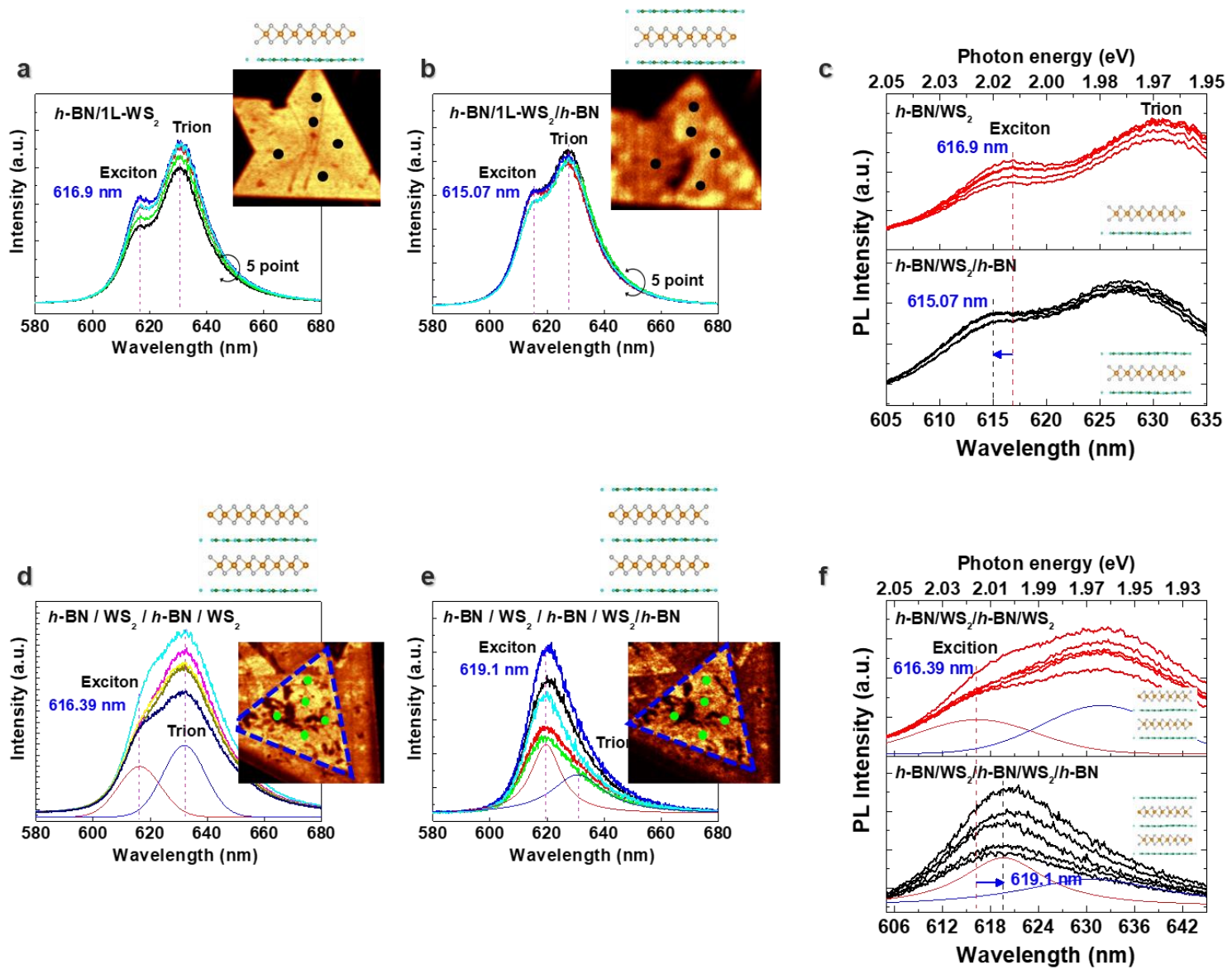

Figure S8. Detailed PL characterizations and Lorentian fitting of trion and exciton peaksin pre-stacked and stacked QW structures. Representative PL spectra, collected from five different points on the transferred $\mathrm{hBN} / \mathrm{WS}_{2}$ (a) pre-stacked structure, before placing a topmost $\mathrm{hBN}$ layer (average exciton wavelength, $616.9 \mathrm{~nm}$ ), and (b) single-stacked $\mathrm{hBN} / \mathrm{WS}_{2} \mathrm{QW}$, after placing the topmost hBN layer (average exciton wavelength, $615.07 \mathrm{~nm}$ ). (c) Exciton energy blue shift occurs after placing the topmost hBN layer. PL spectra, collected from five different points on the transferred $\mathrm{hBN}-\mathrm{WS}_{2}$ (d) pre-stacked structure for double-stacked structure, before placing a topmost hBN layer (average exciton wavelength, $616.39 \mathrm{~nm}$ ), and (e) doublestacked $\mathrm{hBN} / \mathrm{WS}_{2} \mathrm{QWs}$, after placing the topmost $\mathrm{hBN}$ layer (average exciton wavelength, $619.1 \mathrm{~nm}$ ). (f) Exciton energy red shift occurs after placing the topmost hBN layer. The fitted exciton and trion peak values were consistent across different measured points. 
Table S1. Average value of exciton and trion peak positions from Figure S8c

\begin{tabular}{|c|c|c|c|c|c|c|}
\hline Position & 1 & 2 & 3 & 4 & 5 & Average \\
\hline Exciton (nm) & 616.63 & 616.67 & 617.25 & 617.17 & 617.03 & $\begin{array}{c}616.9 \mathbf{~ n m} \\
(\mathbf{E r} \pm \mathbf{0 . 2 5} \mathbf{n m})\end{array}$ \\
\hline Trion (nm) & 615 & 614.83 & 615.16 & 615.33 & 615.16 & $\begin{array}{c}\mathbf{6 1 5 . 0 9} \mathbf{n m} \\
\mathbf{( E r \pm 0 . 1 7 )}\end{array}$ \\
\hline
\end{tabular}
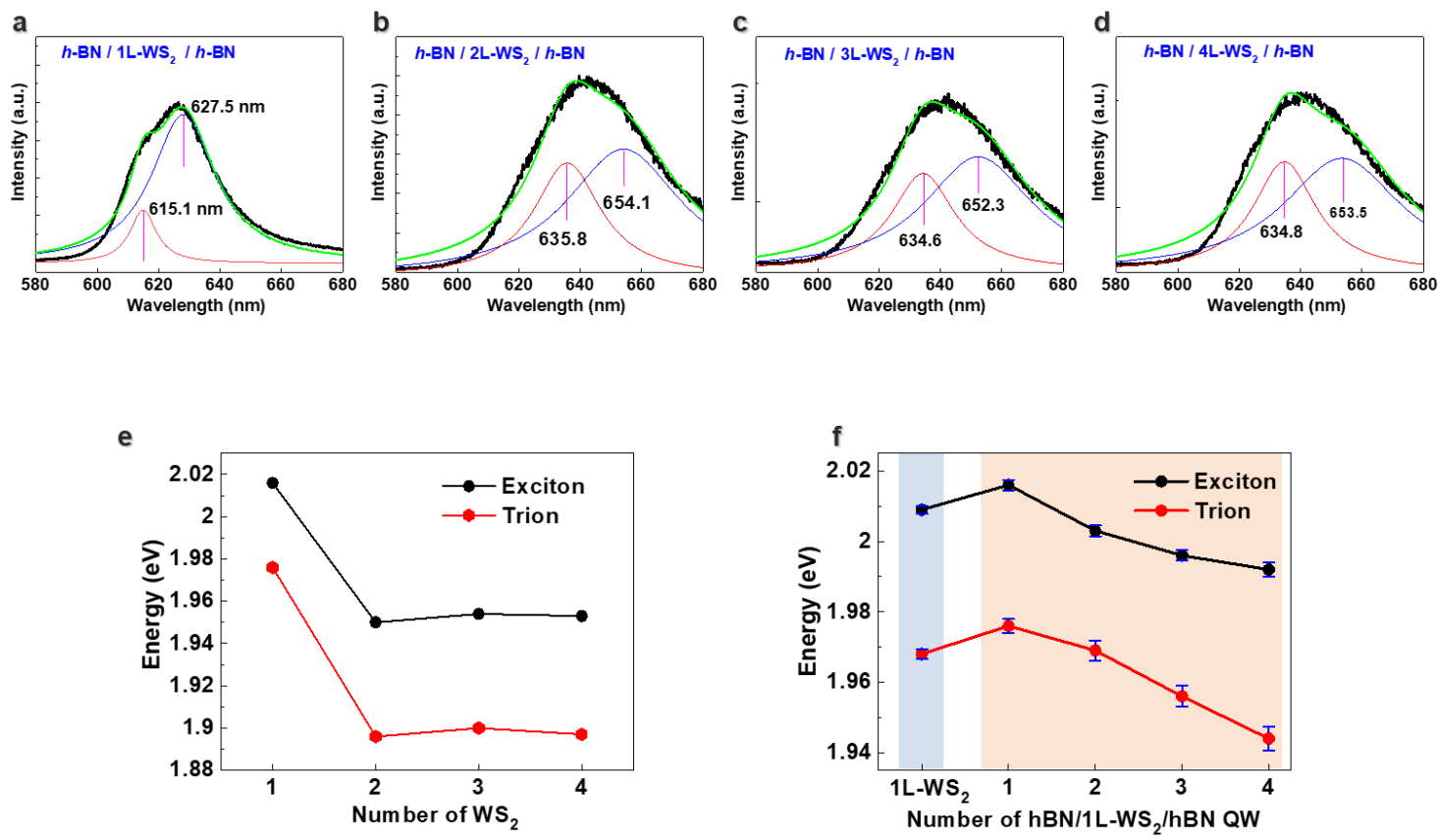

Structure :
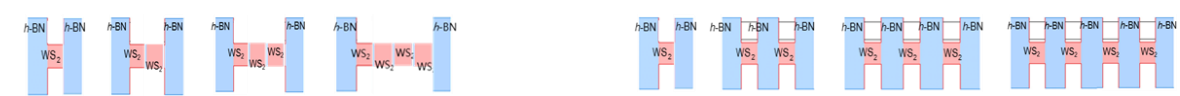

Figure S9. (a-d) PL spectra of the hBN/1 or 2 or 3 or $4 \mathrm{~L}-\mathrm{WS}_{2} / \mathrm{hBN}$ structures. Exciton and trion energy plotted by the number of stacked $\mathrm{WS}_{2}$ layers (e) and the number of stacked $\mathrm{hBN} / 1 \mathrm{~L}-\mathrm{WS}_{2} / \mathrm{hBN} \mathrm{QW}$ layers (f). 


\section{Transfer Process of 2D materials}

\section{WS $_{2}$ Transfer Steps/Process}

1. 495 PMMA A resists $1000 \mathrm{rpm}$ for $10 \mathrm{sec}$ followed by $4000 \mathrm{rpm}$ for $50 \mathrm{sec}$ for $500 \mathrm{~nm}$ thickness PMMA on the 300-nm thick $\mathrm{SiO}_{2} / \mathrm{Si}$ substrate. With a hot plate bake at $180 \sim 200{ }^{\circ} \mathrm{C}$ for 2 minutes.

2. Separating of PMMA $+\mathrm{WS}_{2}$ layer from $\mathrm{Si}$ sub. after removing $\mathrm{SiO}_{2}$ in $10 \% \mathrm{HF}$ solution.

3. Pick and place on the $\mathrm{SiO}_{2} / \mathrm{Si}$ substrate with pre-transferred 2D layer.

\section{hBN transfer Steps/Process}

1. 495 PMMA A resists $1000 \mathrm{rpm}$ for $10 \mathrm{sec}$ followed by $4000 \mathrm{rpm}$ for $50 \mathrm{secs}$ for $500 \mathrm{~nm}$ thickness PMMA. With a hot plate bake at $180 \sim 200{ }^{\circ} \mathrm{C}$ for 2 minute.

2. Separating of PMMA $+\mathrm{hBN}$ layer by using $\mathrm{H}_{2}$ bubbling in $0.1 \mathrm{M} \mathrm{NaOH}$ solution with $10 \mathrm{~V}$ bias on the Pt electrode.

\section{a, $\mathrm{WS}_{2}$ transfer \\ 1. Spin coating of PMMA}

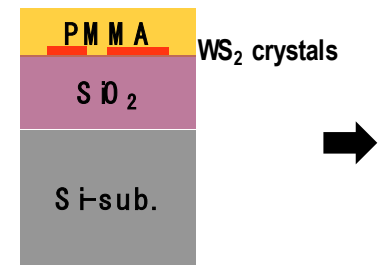

b, hBN transfer
2. $\mathrm{SiO}_{2}$-etching with $\mathrm{HF} /$ removing PMMA

\section{PM M A}

$\mathrm{SiO}_{2}$ etching

Sirsub.
3. Deterministic transfer by using manual transfer system $\begin{array}{ll}\text { 1. Spin coating of PMMA } & \text { 2. Cu-foil separating }\end{array}$ by using $\mathrm{H}_{2}$ bubbling

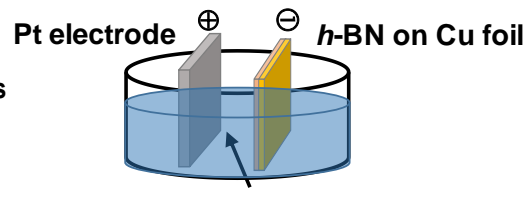

3. Deterministic transfer of hBN

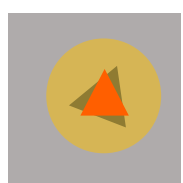

Figure S10. Transfer process flow of the $\mathrm{WS}_{2}$ and $\mathrm{hBN}$ for stacked structure. The process illustrates two different transfer processes for $\mathrm{WS}_{2}$ and $\mathrm{hBN}$. For (a) $\mathrm{WS}_{2}$ transfer and (a) hBN transfer 